\title{
Preliminary phytochemical screening, FTIR and GC-MS analyses of aqueous, ethanolic and methanolic extracts of stem of Tinospora cordifolia (Willd.) Miers for search of antidiabetic compounds
}

Preety Dubey, Shiv Kumar Jayant and Nalini Srivastava

School of Studies in Biochemistry, Jiwaji University, Gwalior-474011, Madhya Pradesh, India

\section{Article Info}

Article history

Received 11 October 2020

Revised 26 November 2020

Accepted 29 November 2020

Published online 30 December 2020

\section{Keywords}

FTIR

GC-MS

Herbal medicines

Tinospora cordifolia (Willd.) Miers

Phytochemicals

\begin{abstract}
Phytochemicals present in different medicinal plants have shown immense therapeutic potential against number of diseases including diabetes. Tinospora cordifolia (Willd.) Miers, a well known medicinal plant, has a high medicinal importance. The study is aimed to fractionate, identify and compile the structure and medicinal properties of each phytochemical of $T$. cordifolia from the available literature, so that the compounds with antioxidant and antihyperglycemic potential can be studied further using in vivo system. The extracts of $T$. cordifolia, prepared in water, ethanol and methanol, were fractionated and the phytochemicals were identified by FTIR and GC-MS. The structure and properties of each component were compiled from the available literature, so that the compounds with antioxidant and antihyperglycemic effects could be studied further. Qualitative tests for the presence of carbohydrates, proteins, tannins, phenolics, glycosides, alkaloids and flavanoids were performed in extracts of $T$. cordifolia. The peaks obtained in FTIR spectra of various extracts of $T$. cordifolia were identified for presence of different functional groups. The phytocomponents resolved by GC-MS in extracts of T. cordifolia are known to contribute to its antioxidant, cancer-preventive, hypercholesterolemic, nematicidal, antifungal, antidiabetic, and hepatoprotective effects. Highest amounts of proteins, tannins, phenol and flavanoids were extracted in water while carbohydrates and glycosides were maximally present in methanolic extract and glycoside in ethanolic extract. Comparative phytochemical analysis of aqueous, ethanolic and methanolic extracts of $T$. cordifolia showed differential distribution of antioxidant and antidiabetic compounds. Further validation of these effects under in vivo system may give leads for the development of herbal alternative for diabetes.
\end{abstract}

\section{Introduction}

Plants play a very important role in maintaining the health of communities and individuals in general and have found medicinal usage in folk and other systems of medicine since time immemorial (Ahn, 2017; Pan et al., 2014). Approximately 3-4 billion people living in developing countries depend on traditional herbal medicines which represent approximately $88 \%$ of the world's population who rely for their primary healthcare on traditional medicines (Jaiswal and Williams, 2016; Vaidya and Devasagayam, 2007). World Health Organization (WHO) has defined medicinal plant as any type of plant whose one or more parts are used for therapeutic and/ or prophylactic purpose (WHO, 2005). The development of traditional herbal medicines has been influenced by different cultural and geographical locations where they were developed first. The major concern in development of herbal medicine is the holistic

Corresponding author: Dr. Nalini Srivastava Professor and Head, School of Studies in Biochemistry, Jiwaji University, Gwalior-474011, Madhya Pradesh, India

E-mail: nalinis21@yahoo.com

Tel.: +91-9425335703

Copyright (c) 2020 Ukaaz Publications. All rights reserved.

Email: ukaaz@yahoo.com; Website: www.ukaazpublications.com approach to life, maintaining balance between mind, body and the environment with emphasis on the good health rather than prevention or cure of diseases (Karmi et al., 2015; Ekor, 2014). Natural products, such as plant extracts, either as standardized therapeutic regimen or isolated pure compound provides ultimate opportunity for discovery of new drugs because of unmatched chemical diversity, affordable cost and ease of availability (Sofowora et al., 2013). The phytochemical analysis of $T$. cordifolia plant is important because of its immense medicinal value against number of ailments, thus great interests of the pharmaceutical companies for the development of new formulations with therapeutic potential against various diseases.

There are many different systems of traditional medicine known, all of which were aimed on maintaining the health rather than curing a disease. However, due to spread of various diseases, the focus of medical practitioners and researchers had been shifted towards search of herbal remedies for cure of such diseases and all efforts of extensive research have been diverted in new direction. The welfare of mankind is possible only when well-developed efficient medical system of cure and control of various diseases is easily available to the common people and masses. Herbal medicines provide most 
economic, easily available and safe system of cure of various diseases that it is in practice since ancient times and has progressively been enriched by research and learned practitioners. Benefits of ancient systems of traditional medicine are well recognized in present era. Lots of drugs used in modern medicine have derived their origin from plant sources (NMPB, 2016; Mosihuzzamn, 2012; Balunas and Kinghom, 2005; Rates, 2001; Balandrin et al., 1985). T. cordifolia stands out as an exceptional herb with a multitude of medicinal benefits (Saha and Ghosh, 2012), belongs to the family, Menispermaceae, which is easily grown in different varieties of soil ranging from acidic to alkaline and requires humidity (Albinjose et al., 2015). Guduchi, the Sanskrit name of $T$. cordifolia, means one which protects the entire body; another term amrita is attributed to its ability to impart vitality, longevity and youthfulness (Dhama et al., 2017). The leaves afford a good fodder for cattle. T. cordifolia is recommended for fever, edema, burning sensation, diabetes, general immunostimulant, anemia, skin diseases and hepatoprotection, etc. (Kaur et al., 2009). T. cordifolia is a plant which has been scientifically validated in various animal models for antioxidant, anti-inflammatory, immunomodulatory, hypoglycemic and other pharmacological activities (Panchabhai et al., 2008; Adhvaryu et al., 2007; Zhao et al., 1991). T. cordifolia is used for treatment of different ailments as well as general tonic for prevention of illness by maintenance of balance between body, mind and environment rather than responding to symptoms of diseases. In our previous studies, the extracts of stem of $T$. cordifolia prepared in water, ethanol and methanol showed antihyperglycemic, antioxidant and hepatoprotective activities in rats with experimental diabetes (Dubey and Srivastava, 2017). Despite very extensive studied plant due to its medicinal value, detailed literature search has shown some gaps in the phytochemical analysis with special focus on antidiabetic and antioxidant compounds in T. cordifolia. The present study is aimed to extract the phytoconstituents in water, ethanol and methanol according to their polarity and their identification by fourier transform infrared spectroscopy (FTIR) and gas chromatography-mass spectroscopy (GC-MS), both techniques are very powerful analytical tools for fractionation and identification of complex mixtures. Attempts have also been made to identify the constituents extracted in water, ethanol and methanol by qualitative phytochemical tests and to collect and compile the information about the medicinal properties of isolated phytocomponents for future validation.

\section{Materials and Methods}

\subsection{Collection of T. cordifolia stem and preparation of extracts}

The dried stem of $T$. cordifolia were purchased from the local herbal market (identified and authenticated by CSIR-National Institute of Science Communication and Information Resources, New Delhi, Botanist: Dr. Sunita Garg, Voucher Specimen Number: NISCAIR/ RHMD/3415-16), dried in shade and powdered with the help of mixer grinder. The extract of $T$. cordifolia was prepared by the method of Khan and Srivastava (Khan and Srivastava, 2012). Separately $30 \mathrm{~g}$ stem powder of $T$. cordifolia was suspended in $100 \mathrm{ml}$ of water, ethanol and methanol, kept on magnetic stirrer for $1 \mathrm{~h}$ and incubated at $5^{\circ} \mathrm{C}$ in a refrigerator for $24 \mathrm{~h}$. Next day, the extracts were placed on magnetic stirrer for $1 \mathrm{~h}$, filtered with Whatman filter paper (Grade 2) and the filtrates were dried and the powder obtained was used in the study.

\subsection{Phytochemical screening}

The phytocomponents present in the extracts of the stem of $T$. cordifolia prepared in water, ethanol and methanol, were qualitatively analyzed for the presence of carbohydrates, protein, tannins and phenol, glycosides, flavonoids and alkaloids in detail as per the standard protocols (Lee et al., 2013; Dhandapani and Babna, 2008). Molisch's test was performed by the method described by Foulger (1931), while other tests of carbohydrates were performed by standard procedures. Qualitative test for proteins was performed by the Biuret method (https://en.wikipedia.org/ wiki/Biuret_test) while ninhydrin test was performed by the method described by Robert (1965). Other phytochemicals were detected by the method described by Ezeonu and Ejikene (2016).

\subsection{Fourier transform infrared spectroscopic (FTIR) analysis}

Fourier transform infrared spectroscopic (FTIR) analysis of the extracts was carried out by the method of Tao et al. (2015), using Spectrum II, Perkin Elmer FTIR. Aqueous, ethanolic and methanolic extracts of $T$. cordifolia were oven dried to get powders of the different solvent extracts to be used for FTIR analyses. Approximately, $10 \mathrm{mg}$ dried extracts powder was used to prepare translucent sample disc by KBr pellet method. The method exploits the property that alkali halide becomes plastic when subjected to pressure that form a sheet that is transparent in the IR region. The sample was mixed with $200 \mathrm{mg}$ fine $\mathrm{KBr}$ powder, pulverized and put into disc forming die, dried at $110^{\circ} \mathrm{C}$ for $2-3 \mathrm{~h}$. A force of approximately 8 tons was applied under vacuum, degassing was performed to remove air and moisture from KBr. Analysis of the sample- $\mathrm{KBr}$ disc was carried out by scanning the samples in the wave number range of 400 to $4000 \mathrm{~cm}^{-1}$ with a resolution of $2 \mathrm{~cm}^{-1}$.

\subsection{Gas chromatography-Mass spectrometry}

\subsubsection{Preparation of sample for GC-MS}

The powder obtained after extraction of $30 \mathrm{~g}$ dried stem of T. cordifolia in water, ethanol and methanol, was dissolved in $2 \mathrm{ml}$ methanol, $100 \mu \mathrm{l}$ sample was taken and dissolved in $1300 \mu \mathrm{l}$ methanol (dilution 1:14) and was used for GC-MS analysis.

GC-MS analysis was carried out by the method described by Adeoye-Isijola et al. using the fused silica capillary column composed of dimethyl poly siloxane, and helium as carrier gas at a constant flow rate of $1 \mathrm{ml} / \mathrm{min}$ (Adeoye-Isijola et al., 2018). The eluted components were detected in the mass detector.

\section{Results}

The herbal medicines are gradually gaining popularity due to their effectiveness, low cost, ease of availability and above all minimum or no side effects when compared with synthetic chemical drugs. Present study is carried out to screen the phytochemicals present in stem of T. cordifolia using different solvents, viz., water, ethanol and methanol, in the light of their antidiabetic and antioxidant properties. 
3.1 Qualitative phytochemical analysis of the extracts of T. cordifolia stem prepared in water, ethanol and methanol

The results of qualitative phytochemical analyses of aqueous, ethanolic and methanolic extracts of stem of T. cordifolia are given in Table 1 and Figure 1. All the three extracts tested for different phytochemicals showed the presence of alkaloids, flavonoids, glycosides, carbohydrates, proteins, tannins and phenols. The results presented in Table 1 clearly showed that all the three solvents are equally efficient in extracting carbohydrates while reducing sugars were maximally extracted in methanol; ketoses in water and pentoses in water and methanol equally. Water seems to be the best solvent for proteins and peptides, followed by ethanol while methanolic extract has not shown the presence of proteins tested by biuret method; the amino acids have been extracted equally in all the three solvents. Glycosides were maximally extracted in methanol while flavonoids were extracted only in aqueous extract. Alkaloids were maximally extracted in ethanolic fraction followed by methanol and water (Table 1).

\subsection{FTIR-analysis}

FTIR is the most powerful technique to identify the functional groups present in different extracts of $T$. cordifolia. The absorption maxima in the IR spectrum help to identify the functional groups of the active components present in different extracts (Maobe and Nyarango, 2013). When the extracts were analyzed into the
FTIR, the different functional groups of the components were separated and were shown as peaks in the FTIR spectra. FTIR analysis of all the three extracts of the stem of T. cordifolia is presented in Table 2 and Figure 2. The results of FTIR analysis confirmed the presence of alcohol, aldehyde, alkyne, alkene, amines and ester. The absorbance bands analysis in bioreduction process are observed in the region between $400-4000 \mathrm{~cm}^{-1}$.

\subsection{GC-MS analysis}

For fractionation of aqueous, ethanolic and methanolic extracts of $T$. cordifolia, a powerful separation technique, gas chromatography combined with powerful identification technique of mass spectroscopy (GC-MS) is used. GC-MS analysis of all the three extracts of the stem of $T$. cordifolia is presented in Table 3 and in Figure 3. In the GC-MS analysis of various extracts of T. cordifolia stem, a total of 65 components have been detected. Individually, aqueous extract contained 15 compounds, ethanolic extract contained 18 compounds, and methanolic extract contained 32 compounds (Table 3). Beta amyrin, androstan-17-one, and 3-ethyl-3-hydroxy- (5. Alpha) are common compounds present in all three extracts of $T$. cordifolia, however maximum amount of beta amyrin is extracted in aqueous, followed by ethanolic and methanolic extracts approximated by the obtained peak areas $(2.64,2.13$ and 2.11 in aqueous, ethanolic and methanolic extracts, respectively Tabel 4). Medicinal properties of each components isolated and identified by GS-MS have been compiled on the basis of available information (Table 5).

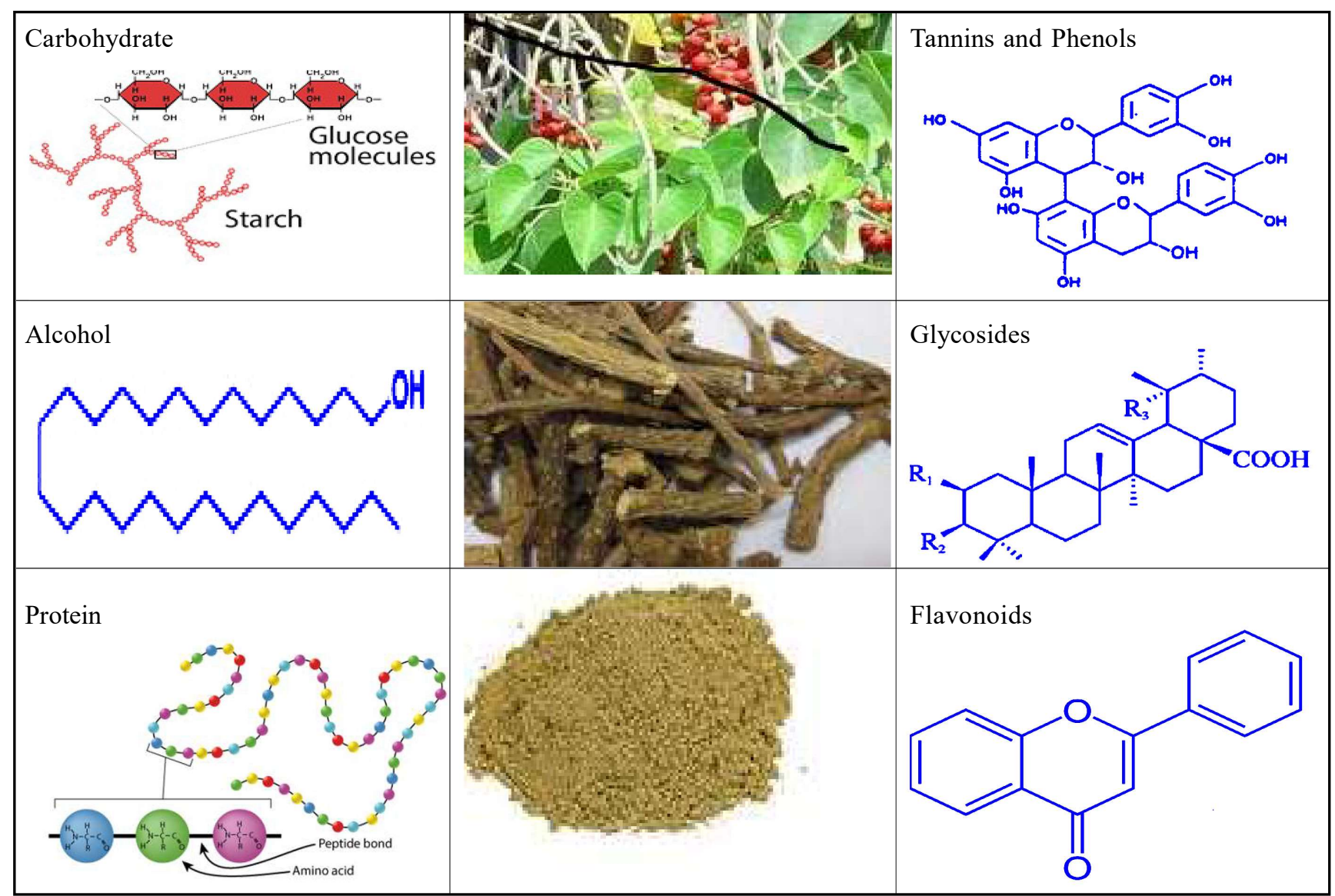

Figure 1: Tinospora cordifolia: Plant, dried stem and its powder. 


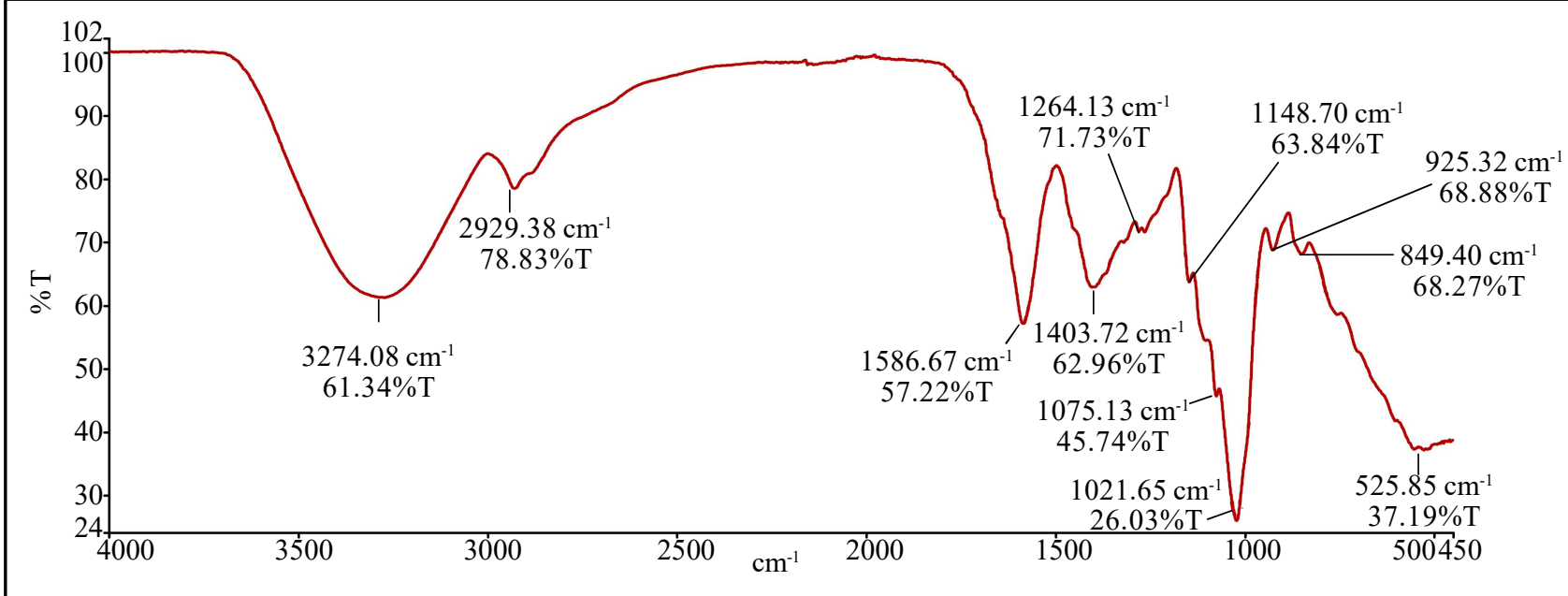

2A: (Aqueous extract)

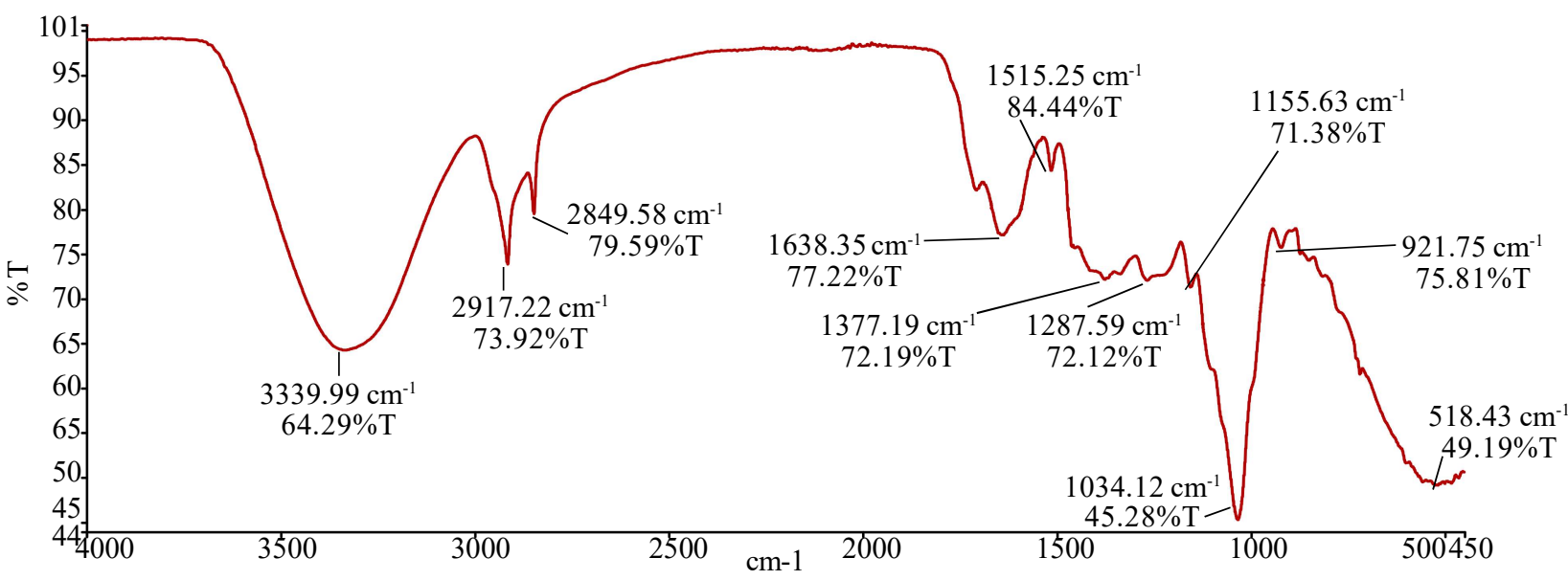

2B: (Ethanolic extract)

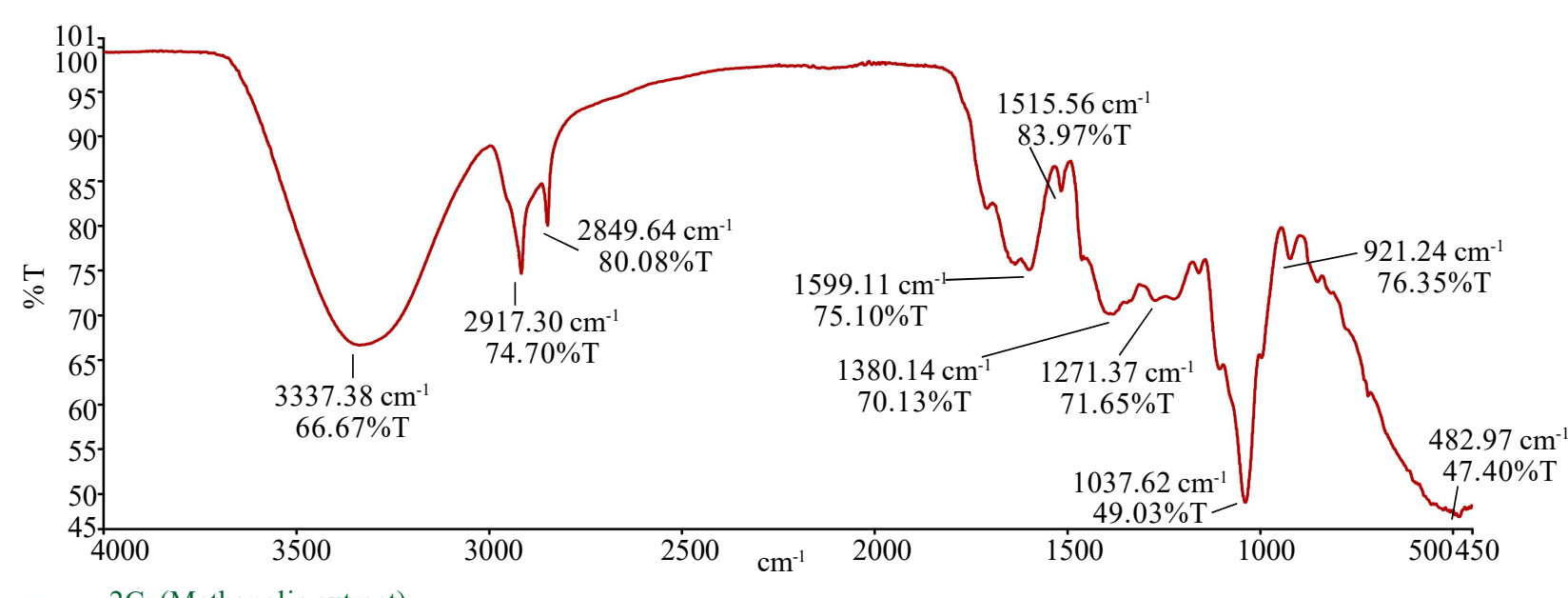

2C: (Methanolic extract)

Figure 2: FTIR Chromatogram of aqueous, ethanolic and methanolic extract of $T$. cordifolia. 


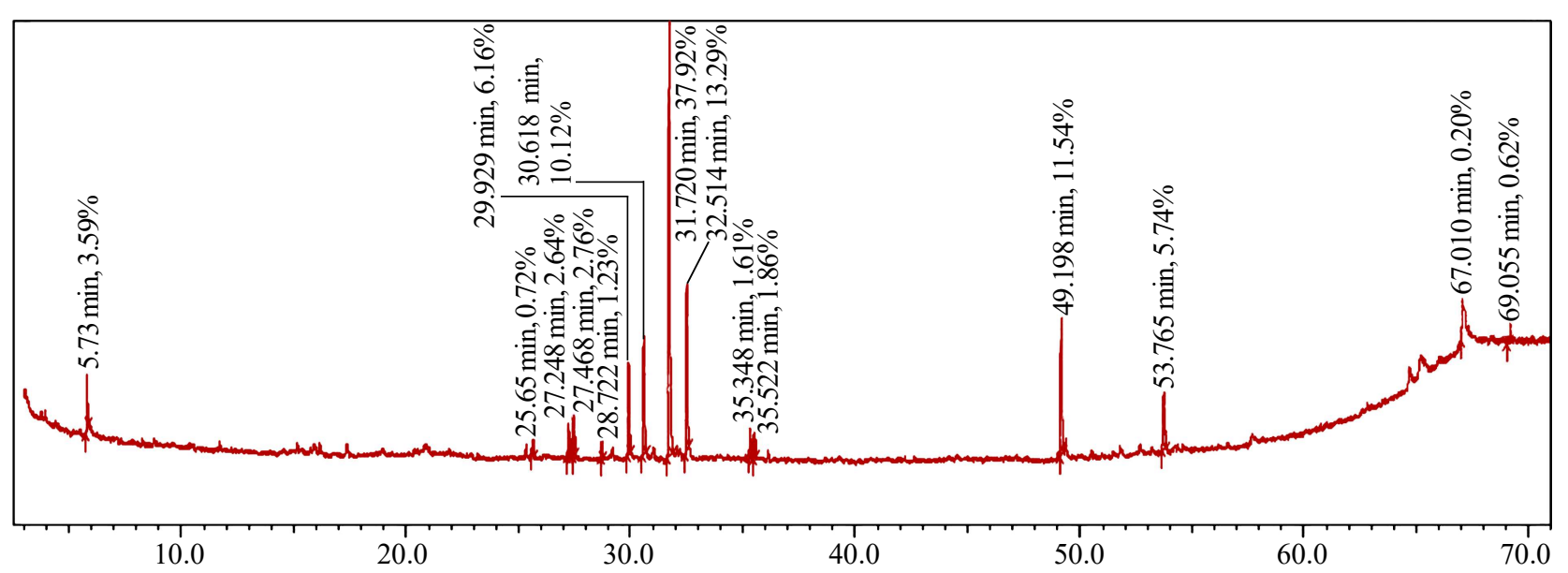

3A: GC-MS chromatogram of aqueous extract

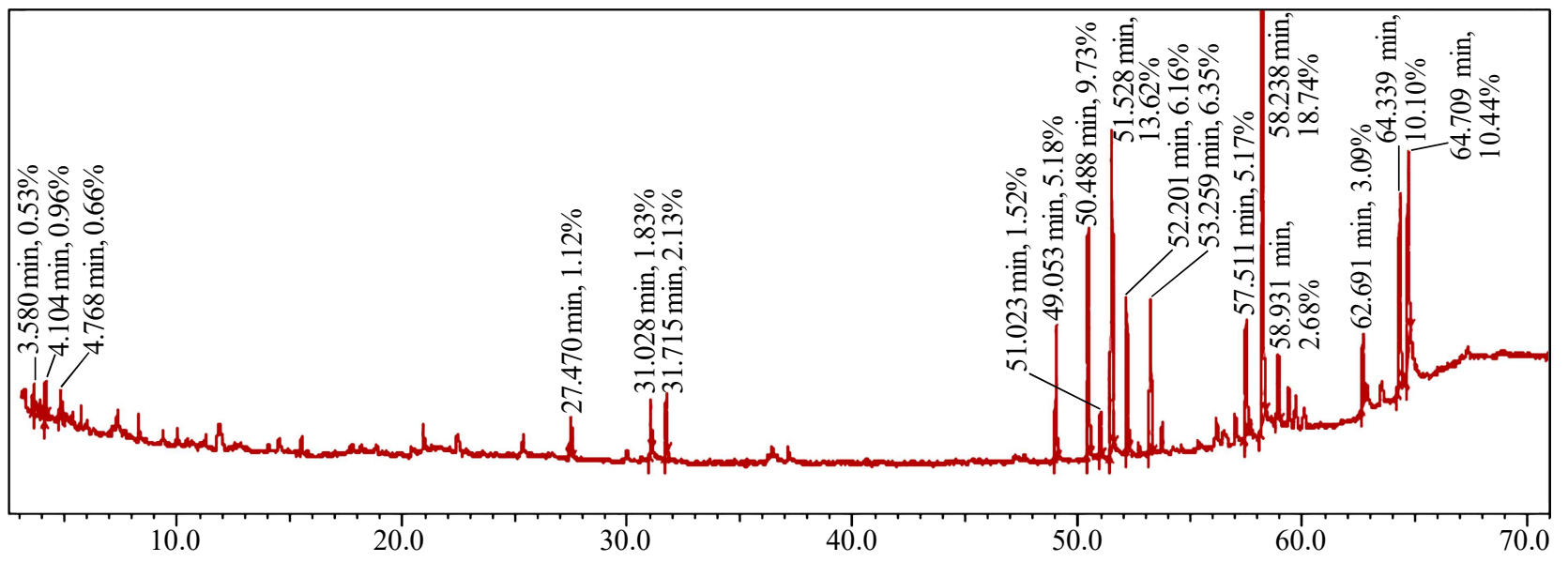

3B: GC-MS Chromatogram of ethanolic extract

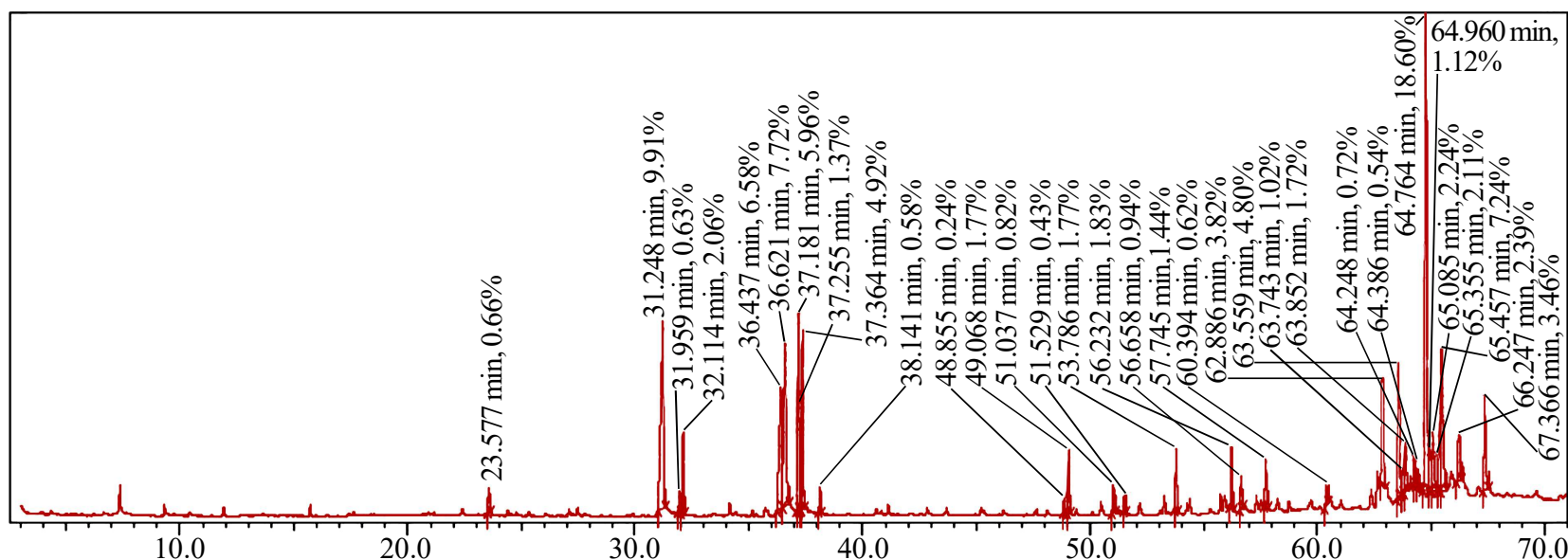

3C: GC-MS Chromatogram of methanolic extract

Figure 3: GC-MS Chromatogram of aqueous, ethanolic and methanolic extracts of $T$. codifolia. 
Table 1: Qualitative phytochemical screening of aqueous, ethanolic and methanolic extracts of the stem of T. cordifolia

\begin{tabular}{|c|c|c|c|c|c|c|c|}
\hline Constituents & Test & \multicolumn{6}{|c|}{ Results } \\
\hline \multirow{6}{*}{ Carbohydrates } & \multirow{3}{*}{$\begin{array}{l}\text { Molisch's test } \\
\text { Fehling's test }\end{array}$} & Negative control & Std $(0.1 \%)$ & Std $(1 \%)$ & Aqueous extract & Ethanolic extract & Methanolic ext. \\
\hline & & - & ++ & +++ & +++ & +++ & +++ \\
\hline & & - & ++ & +++ & - & ++ & +++ \\
\hline & Benedict's test & - & + & +++ & +++ & ++ & +++ \\
\hline & Seliwanoff's test & - & + & +++ & +++ & + & ++ \\
\hline & Bial's test & - & ++ & +++ & +++ & ++ & +++ \\
\hline \multirow[t]{2}{*}{ Protein } & Biuret's test & - & + & +++ & +++ & + & - \\
\hline & Ninhydrin's test & - & + & +++ & +++ & + & +++ \\
\hline \multirow[t]{2}{*}{ Tannin and phenol's } & Ferric chloride test & - & +++ & +++ & ++ & + & ++ \\
\hline & Lead acetate test & - & + & +++ & +++ & + & ++ \\
\hline \multirow[t]{3}{*}{ Glycosides } & Keller-Killani test & - & ++ & +++ & +++ & +++ & +++ \\
\hline & Borntrager's test & - & ++ & +++ & ++ & + & + \\
\hline & Legal's test & - & ++ & +++ & - & + & ++ \\
\hline Flavanoids & Shinoda's test & - & + & +++ & ++ & - & - \\
\hline \multirow[t]{4}{*}{ Alkaloid's test } & Wagner's test & - & ++ & +++ & +++ & +++ & +++ \\
\hline & Hager's test & - & + & +++ & + & +++ & ++ \\
\hline & Mayer's test & - & + & +++ & + & +++ & +++ \\
\hline & Dragendroff's test & - & ++ & +++ & +++ & +++ & +++ \\
\hline
\end{tabular}

- Signs:- absent; + slight presence; ++ moderate color; +++ intense color

- Standards used for carbohydrate: Glucose for Molish's, Fehling's and Benedict test; fructose for Selwinoff's test and xylose for Bial's test; for protein: bovine serum albumin for Biuret's and ninhydrin's tests; for tannins and phenol: catechol for ferric chloride and lead acetate test; for glycosides: sucrose for Keller- Killani, Borntrager's and Legal's tests; for flavanoids: for Shinoda's test standard used as quercetin; and for alkaloids: caffeine was used as standard Wagner's, Hager's, Mayer's and Dragendroff's tests.

- Each test was performed three times and the results were complied on the basis of intensity of color observed.

Table 2: Peaks observed in FTIR spectra of aqueous, ethanolic and methanolic extracts of $T$. cordifolia and corresponding probable functional group

\begin{tabular}{|l|c|l|l|l|l|l|}
\hline & \multicolumn{2}{|c|}{ Aqueous extract } & \multicolumn{2}{c|}{ Ethanolic extract } & \multicolumn{2}{c|}{ Methanolic extract } \\
\hline S.No. & $\begin{array}{l}\text { Observed peak } \\
\left.\text { (wave number } \mathrm{cm}^{-1}\right)\end{array}$ & Functional groups & $\begin{array}{l}\text { Observed peak } \\
\left(\text { wave number } \mathrm{cm}^{-1}\right)\end{array}$ & Functional groups & $\begin{array}{l}\text { Observed peak } \\
\left(\text { wave number } \mathrm{cm}^{-1}\right)\end{array}$ & Functional groups \\
\hline 1 & 3274.08 & Hydroxyl compound & 3339.99 & Hydroxyl compound & 3337.38 & Hydroxyl compound \\
2 & 2929.38 & Alkene & 2917.22 & Methyl group & 2917.30 & Methyl group \\
3 & 1586.67 & Aromatic & 2849.58 & Alkane & 2849.64 & Alkane \\
4 & 1403.72 & Nitrosamine & 1638.35 & Conjugated Alkene & 1599.11 & Aromatic ring \\
5 & 1264.13 & Alcohols, Esters & 1515.25 & Phenol ring & 1515.56 & Phenol ring \\
6 & 1146.70 & Aliphatic ether & 1377.19 & Phenol & 1380.14 & Phenol \\
7 & 1075.13 & Amines & 1287.59 & Ester & 1271.37 & Aromatic ester \\
8 & 1021.65 & Alkyl amine & 1034.12 & Amines & 921.24 & Amines \\
9 & 925.32 & Ether & 1155.63 & Ester & 487.97 & Halogen compound \\
10 & 849.40 & Alkene & 921.75 & Ether & - \\
\hline 11 & 525.85 & Halogen compound & 518.43 & Halogen compound & - \\
\hline
\end{tabular}

- The FTIR of Perkin Elmer Spectrum II, was used for the present study.

- IR spectra of aqueous, ethanolic and methanolic extract of T. cordifolia were scanned as function of \% Transmittance versus wave number $\left(\mathrm{cm}^{-1}\right)$.

- The functional groups giving the particular peak were identified from the reported values.

- The experiment was repeated twice.

Table 3: Phytochemicals resolved by GC-MS in aqueous, ethanolic and methanolic extracts of T. cordifolia

\begin{tabular}{|c|c|c|c|c|c|c|c|}
\hline \multicolumn{2}{|c|}{ S.No. Name of the compound } & \multicolumn{2}{|c|}{ Aqueous extract } & \multicolumn{2}{|c|}{ Ethanolic extract } & \multicolumn{2}{|c|}{ Methanolic extract } \\
\hline & & Rt (min) & PA $(\%)$ & Rt (min) & PA $(\%)$ & Rt (min) & PA $(\%)$ \\
\hline 1. & 2-Piperidinone & 5.73 & 3.59 & - & - & - & - \\
\hline 2. & Tridecanoic acid,12-methyl-, methyl ester & 25.65 & 0.72 & - & - & - & - \\
\hline 3. & Beta amyrin & 27.248 & 2.64 & - & - & - & - \\
\hline 4. & Calcitriol & 27.468 & 2.76 & - & - & - & - \\
\hline 5. & Ethyl tridecanoate & 28.722 & 1.23 & - & - & - & - \\
\hline 6. & Hexadecanoic acid, methyl ester & 29.929 & 6.16 & - & - & - & - \\
\hline 7. & Androstan-17-one, 3-ethyl-3-hydroxy-, (5. Alpha & 30.618 & 10.12 & - & - & - & - \\
\hline 8. & 2,6,8-Trimethylbicyclo[4.2.0]oct-2-ene-1,8dio- & 31.720 & 37.92 & - & - & - & - \\
\hline 9. & 1,Z-5,E-7-Dodecatriene & 32.514 & 13.29 & - & - & - & - \\
\hline
\end{tabular}




\begin{tabular}{|c|c|c|c|c|c|c|c|}
\hline \multirow[t]{2}{*}{ S.No. } & Name of the compound & \multicolumn{2}{|c|}{ Aqueous extract } & \multicolumn{2}{|c|}{ Ethanolic extract } & \multicolumn{2}{|c|}{ Methanolic extract } \\
\hline & & Rt (min) & PA $(\%)$ & Rt (min) & PA $(\%)$ & Rt (min) & PA $(\%)$ \\
\hline 10. & 8-Azabicyclo[3,2,1]octan-3-ol, 8-methyl-endo- & 35.348 & 1.61 & - & - & - & - \\
\hline 11. & 7-Hexadecenoic acid, methyl ester, $(\mathrm{Z})$ & 35.522 & 1.86 & - & - & - & - \\
\hline 12. & Alfa-Copaene & 49.198 & 11.54 & - & - & - & - \\
\hline 13. & 1'-Vinyl-1,-2;-3; -4tetrahydrospiro[1,3]dioxolar & 53.765 & 5.74 & - & - & - & - \\
\hline 14. & Phthalic acid & 67.010 & 0.20 & - & - & - & - \\
\hline 15. & Heptacosanol & 69.055 & 0.62 & - & - & - & - \\
\hline 16. & 1-Butanamine, 3-methyl-N-(3-methylbutalide & - & - & 3.580 & 0.53 & - & - \\
\hline 17. & Butanedioicacid,monomethyl ester & - & - & 4.104 & 0.96 & - & - \\
\hline 18. & 1-(+)-Lactic acid, tert-butyldimethylsilyl ester & - & - & 4.768 & 0.66 & - & - \\
\hline 19. & 5-Isopropylidene-4, 6-dimethylnona-3,6,8 trien- & - & - & 27.470 & 1.12 & - & - \\
\hline 20. & Pentadecanoic acid & - & - & 31.028 & 1.83 & - & - \\
\hline 21. & Beta amyrin & - & - & 31.715 & 2.13 & - & - \\
\hline 22. & 1H-3a,7-Methanoazulene, octahydro-1,9,9-trimethyl- & - & - & 49.053 & 5.18 & - & - \\
\hline 23. & 2,2,6beta, 7-Tetramethylbicyclo[4,3,0]nona-1 & - & - & 50.488 & 9.73 & - & - \\
\hline 24. & $\begin{array}{l}\text { 1,4-Methanocycloocta[d]pyridazine, } 1,4,4 \mathrm{a}, 5,6,9,10,10 \mathrm{a}- \\
\text { octahydro-11,11-Dimethyl }\end{array}$ & - & - & 51.023 & 1.52 & - & - \\
\hline 25. & Androstan-17-one, 3-ethyl-3-hydroxy-,(5. Alpha) & - & - & 51.528 & 13.62 & - & - \\
\hline 26. & 3-Amino-6-methyl-6,7-dihydro-9H-5-oxa-9-aza & - & - & 52.201 & 6.16 & - & - \\
\hline 27. & Azulene, $1,2,3,5,6,7,8,8 \mathrm{a}$-octahydro-1,4-dimethyl & - & - & 53.259 & 6.35 & - & - \\
\hline 28. & Cyclopentanecarboxamide, 2-[1-(2-butenyl)-3- & - & - & 57.511 & 5.17 & - & - \\
\hline 29. & Beta-Vatirenene & - & - & 58.238 & 18.74 & - & - \\
\hline 30. & 6-Isopropenyl-4,8a-dimethyl-1,2,3,5,6,7,8,8a- & - & - & 58.931 & 2.68 & - & - \\
\hline 31. & (3E,5E,7E)-6-Methyl-8-(2,6,6-trimethyl-1-cyclo & - & - & 62.691 & 3.09 & - & - \\
\hline 32. & Androstan-17-one, 3-ethyl-3-hydroxy-, (5. Al & - & - & 64.339 & 10.10 & - & - \\
\hline 33. & Beta-Sitosterol & - & - & 64.709 & 10.44 & - & - \\
\hline 34. & 4-((1E)-3-Hydroxy-1-propenyl)-2- & - & - & - & - & 23.577 & 0.66 \\
\hline 35. & 1-(+)-Ascorbic acid 2,6-dihexadecanoa & - & - & - & - & 31.248 & 9.91 \\
\hline 36. & Benzenemethanol, 2,5-dimethoxy-, acetate & - & - & - & - & 31.959 & 0.63 \\
\hline 37. & ic acid, methyl ester & - & - & - & - & 32.114 & 2.06 \\
\hline 38. & Ethyl 9,12- hexadecadienoate & - & - & - & - & 36.437 & 6.58 \\
\hline 39. & 9-Octadecenoic acid,1,2,3-propanetriyl ester, (EEE) & - & - & - & - & 36.621 & 7.72 \\
\hline 40. & n-Propyl 9,12-octadecadienoate & - & - & - & - & 37.181 & 5.96 \\
\hline 41. & Octadecanoic acid & - & - & - & - & 37.255 & 1.37 \\
\hline 42. & 9-Octadecanoic acid, 1,2,3-propanetriyl ester, (EEE) & - & - & - & - & 37.364 & 4.92 \\
\hline 43. & Heptadecanoic acid, 15-methyl-, ethyl ester & - & - & - & - & 38.141 & 0.58 \\
\hline 44 & Ethyl tridecanoate & - & - & - & - & 48.855 & 0.24 \\
\hline 45 & 1H-3a, 7-Methanoazulene, octahydro-1,9,9-trimethyl-4-methylene & - & - & - & - & 49.068 & 1.77 \\
\hline 46 & $\begin{array}{l}\text { 1,4- Methanocycloocta[d]pyridazine, } 1,4,4 \mathrm{a}, 5,6,9,10,10 \mathrm{a}- \\
\text { Octahydro-11,11-dimethyl- }\end{array}$ & - & - & - & - & 51.037 & 0.82 \\
\hline 47 & $\begin{array}{l}\text { Cycloprop[e]indene-1a,2(1H)-dimethanol,3a, } 4,5,6,6 \mathrm{a}, 6 \mathrm{~b}- \\
\text { hexahydro-5,5,6b-trimethyl- }\end{array}$ & - & - & - & - & 51.529 & 0.43 \\
\hline 48 & 1-Methoxy-5-methyl-5-phenyl-7-oxabicyclo[ 4. & - & - & - & - & 53.786 & 1.77 \\
\hline 49 & Benzenc & - & - & - & - & 56.232 & 1.83 \\
\hline 50 & , (2-ethyl-4-methyl-1,3-pent & - & - & - & - & 56.658 & 0.94 \\
\hline 51 & Benzene,(2-ethyl-4-methyl-1,3-pentadienyl)-, & - & - & - & - & 57.745 & 1.44 \\
\hline 52 & 1-Heptacosanol & - & - & - & - & 60.394 & 0.62 \\
\hline 53 & 3 beta-Hydroxy-5-cholen-24-oic acid & - & - & - & - & 62.886 & 3.82 \\
\hline 54 & Stigmasterol & - & - & - & - & 63.559 & 4.80 \\
\hline 55. & 1-Tetradecene $n$-tetradec-1-ene & - & - & - & - & 63.743 & 1.02 \\
\hline 56. & (+)- Lariciresinol & - & - & - & - & 63.852 & 1.72 \\
\hline 57. & Obtusifoliol & - & - & - & - & 64.248 & 0.72 \\
\hline 58. & Androstan-17-one, 3-ethyl-3-hydroxy-, (5.alpha) & - & - & - & - & 64.386 & 0.54 \\
\hline 59. & 3.beta-Hydroxy-5-cholen-24-oic acid & - & - & - & - & 64.764 & 18.60 \\
\hline 60. & 5-Isopropylidene-4, 6-dimethylnona-3,6,8-trien- & - & - & - & - & 64.960 & 1.12 \\
\hline 61. & 14- Oxatricyclo[9.2.1.0(1,10)] tetradecane, 2,6,6,10,11-pentamethyl & - & - & - & - & 65.085 & 2.24 \\
\hline 62. & beta-Amyrin & - & - & - & - & 65.355 & 2.11 \\
\hline 63. & 9,19-Cyclo-9.beta-lanostane-3.beta,25-diol & - & - & - & - & 65.457 & 7.24 \\
\hline 64. & $(1 \mathrm{~S}, 6 \mathrm{R}, 9 \mathrm{~S})-5,5,9,10$-Tetramethyltricyclo[7.3.0 & - & - & - & - & 66.247 & 2.39 \\
\hline 65. & 9,19-Cyclolanostan-3-ol,24-methylene-,(3.beta & - & - & - & - & 67.366 & 3.46 \\
\hline
\end{tabular}

- Rt: retention time in min and PA: peak area in \%.

- List of photochemical resolved on the basis of retention time and quantitated on the basis of peak area. 
Table 4: Comparison of compound present in different extracts (aqueous, ethanolic and methanolic) of T. cordifolia stem

\begin{tabular}{|c|c|c|c|c|}
\hline \multirow[t]{2}{*}{ S.No. } & \multirow[t]{2}{*}{ Name of the compound } & \multicolumn{3}{|c|}{ Type of solvent for extraction } \\
\hline & & Water & Ethanol & Methanol \\
\hline 1. & 4-((1E)-3-Hydroxy-1-propenyl)-2-methoxyphenyl & - & - & $P$ \\
\hline 2. & 1-(+)-Ascorbic acid 2,6-dihexadecanoate & - & - & $\mathrm{P}$ \\
\hline 3. & Benzenemethanol, 2,5-dimethoxy-, acetate & - & - & $P$ \\
\hline 4. & Hexadecanoic acid, methyl ester & $\mathrm{p}$ & - & $\mathrm{P}$ \\
\hline 5 . & Ethyl 9,12- hexadecadienoate & - & - & $\mathrm{P}$ \\
\hline 6. & 9-Octadecenoic acid,1,2,3-propanetriyl ester, (EEE) & - & - & $P$ \\
\hline 7. & n-Propyl 9,12-octadecadienoate & - & - & $\mathrm{P}$ \\
\hline 8. & Octadecanoic acid & - & - & $\mathrm{P}$ \\
\hline 9. & Heptadecanoic acid, 15-methyl-, ethyl ester & - & - & $\mathrm{P}$ \\
\hline 10. & Ethyl tridecanoate & $\mathrm{p}$ & & $\mathrm{P}$ \\
\hline 11. & 1H-3a, 7-Methanoazulene, octahydro-1,9,9-trimethyl-4methylene & - & $\mathrm{P}$ & $\mathrm{P}$ \\
\hline 12. & 1,4- Methanocycloocta[d]pyridazine, $1,4,4 \mathrm{a}, 5,6,9,10,10 \mathrm{a}-$ Octahydro- 11,11 dimethyl & - & $\mathrm{p}$ & $\mathrm{P}$ \\
\hline 13. & Cycloprop[e]indene-1 a,2(1H)-dimethanol,3a,4,5,6,6a,6b-hexahydro-5,5,6b-trimethyl & - & - & $\mathrm{P}$ \\
\hline 14. & 1-Methoxy-5-methyl-5-phenyl-7-oxabicyclo[ 4 & - & - & $\mathrm{P}$ \\
\hline 15. & Benzene, 1,2-bis(1-buten-3-yl)- & - & - & $\mathrm{P}$ \\
\hline 16. & Benzene,(2-ethyl-4-methyl-1,3-pentadienyl)-, & - & - & $\mathrm{P}$ \\
\hline 17. & 1-Heptacosanol & $\mathrm{p}$ & - & $\mathrm{P}$ \\
\hline 18. & 3 beta-Hydroxy-5-cholen-24-oic acid & - & - & $\mathrm{P}$ \\
\hline 19. & 1-Tetradecene n-tetradec-1-ene & - & - & $\mathrm{P}$ \\
\hline 20. & $(+)$ - Lariciresinol & - & - & $\mathrm{P}$ \\
\hline 21. & Obtusifoliol & - & - & $\mathrm{P}$ \\
\hline 22. & Androstan-17-one, 3-ethyl-3-hydroxy-, (5.alpha) & $\mathrm{p}$ & $\mathrm{p}$ & $\mathrm{P}$ \\
\hline 23 & 3.beta-Hydroxy-5-cholen-24-oic acid & - & - & $\mathrm{P}$ \\
\hline 24. & 14-Oxatricyclo[9.2.1.0(1,10)]tetradecane, $2,6,6,10,11$-pentamethyl & - & - & - \\
\hline 25. & beta-Amyrin & $\mathrm{P}$ & $\mathrm{p}$ & $\mathrm{P}$ \\
\hline 26 & 9,19-Cyclo-9.beta-lanostane-3.beta,25-diol & - & - & $\mathrm{P}$ \\
\hline 27. & $(1 \mathrm{~S}, 6 \mathrm{R}, 9 \mathrm{~S})-5,5,9,10$-Tetramethyltricyclo[ $[7.3 .0$ & - & - & $\mathrm{P}$ \\
\hline 28. & Piperidinone & $\mathrm{p}$ & - & $\mathrm{P}$ \\
\hline 29. & Tridecanoic acid 12-methyl ester & $\mathrm{P}$ & - & - \\
\hline 30. & Calcitriol & $\mathrm{P}$ & - & - \\
\hline 31. & 2,6,8-Trimethylbicyclo[4.2.0]oct-2-ene-1,8-dio & $\mathrm{p}$ & - & - \\
\hline 32. & 1,Z-5,E-7-Dodecatriene & $\mathrm{p}$ & $\mathrm{p}$ & - \\
\hline 33. & 8-Azabicyclo[3,2,1]octan-3-ol, 8-methyl-, endo- & $\mathrm{P}$ & - & - \\
\hline 34. & Alfa-Copaene & $\mathrm{p}$ & - & - \\
\hline 35. & 1'-Vinyl-1,-2'-3'-4'-tetrahydrospiro[1,3]dioxolar & $\mathrm{P}$ & - & - \\
\hline 36. & Phthalic acid & $\mathrm{p}$ & - & - \\
\hline 37. & 1-Butanamine, 3-methyl-N-(3-methylbutalide & - & $\mathrm{P}$ & - \\
\hline 38. & Butanedioicacid,monomethyl ester & - & $P$ & - \\
\hline 39. & 1-(+)-Lactic acid, tert-butyldimethylsilyl ester & - & $\mathrm{p}$ & - \\
\hline 40. & 5-Isopropylidene-4, 6-dimethylnona-3,6,8-trien- & - & $P$ & - \\
\hline 41. & Pentadecanoic acid & - & $\mathrm{P}$ & - \\
\hline 42. & 3-Amino-6-methyl-6,7-dihydro-9H-5-oxa-9-aza & - & $\mathrm{P}$ & - \\
\hline 43. & Azulene, $1,2,3,5,6,7,8,8 \mathrm{a}$-octahydro-1,4-dimethyl & - & $\mathrm{P}$ & - \\
\hline 44. & Cyclopentanecarboxamide, 2-[1-(2-butenyl)-3- & - & $\mathrm{P}$ & - \\
\hline 45 . & Beta-Vatirenene & - & $P$ & - \\
\hline 46. & 6-Isopropenyl-4,8a-dimethyl-1,2,3,5,6,7,8,8a- & - & $\mathrm{P}$ & - \\
\hline 47. & (3E,5E,7E)-6-Methyl-8-(2,6,6-trimethyl-1-cyclo & - & $P$ & - \\
\hline 48. & Beta-Sitosterol & - & $\mathrm{p}$ & - \\
\hline
\end{tabular}

- Comparison of compounds resolved and identified by GC-MS in different extracts of T. cordifolia.

- P: presence of compound. 
Table 5: Chemical formula, molecular weight and structure of all compounds resolved and identified in aqueous, ethanolic and methanolic extracts of T. cordifolia

\begin{tabular}{|c|c|c|c|c|c|}
\hline S.No & Name of the compound & Structure & $\begin{array}{l}\text { Chemical } \\
\text { formula }\end{array}$ & $\begin{array}{l}\text { Molecular } \\
\text { weight }\end{array}$ & Medicinal properties \\
\hline 1. & $\begin{array}{l}\text { 4-((1E)-3-Hydroxy-1- } \\
\text { propenyl)-2-methoxyphenyl }\end{array}$ & & $\mathrm{C}_{10} \mathrm{H}_{12} \mathrm{O}_{3}$ & 180.2 & $\begin{array}{l}\text { Antimicrobial, } \\
\text { antioxidant, anti- } \\
\text { inflammatory }\end{array}$ \\
\hline 2. & $\begin{array}{l}\text { Benzenemethanol, 2,5- } \\
\text { dimethoxy-, acetate }\end{array}$ & & $\mathrm{C}_{11} \mathrm{H}_{14} \mathrm{O}_{4}$ & 168.2 & Antibacterial activity \\
\hline 3. & Hexadecanoic acid, methyl ester & & $\mathrm{C}_{18} \mathrm{H}_{36} \mathrm{O}_{2}$ & 284.5 & $\begin{array}{l}\text { Antioxidant, pesticide, } \\
\text { hypocholesterolimic }\end{array}$ \\
\hline 4. & Ethyl 9,12- hexadecadienoate & & $\mathrm{C}_{18} \mathrm{H}_{32} \mathrm{O}_{2}$ & 280.5 & $\begin{array}{l}\text { Ntioxidant, pesticide, } \\
\text { hypocholesterolemic }\end{array}$ \\
\hline 5. & $\begin{array}{l}\text { 9-Octadecenoic acid,1,2,3- } \\
\text { propanetriyl ester, (EEE) }\end{array}$ & & $\mathrm{C}_{57} \mathrm{H}_{104} \mathrm{O}_{6}$ & 885.4 & $\begin{array}{l}\text { Antispasmodic, } \\
\text { immunomodulatory }\end{array}$ \\
\hline 6. & n-Propyl 9,12-octadecadienoate & & $\mathrm{C}_{21} \mathrm{H}_{38} \mathrm{O}_{2}$ & 322.5 & $\begin{array}{l}\text { Emulsifying agent, } \\
\text { soaps, paint }\end{array}$ \\
\hline 7. & Octadecanoic acid & & $\mathrm{C}_{18} \mathrm{H}_{36} \mathrm{O}_{2}$ & 284.5 & $\begin{array}{l}\text { Antifungal, Antitumor, } \\
\text { antibacterial }\end{array}$ \\
\hline 8. & $\begin{array}{l}\text { Heptadecanoic acid, } 15 \text { methyl-, } \\
\text { ethyl ester }\end{array}$ & & $\mathrm{C}_{20} \mathrm{H}_{40} \mathrm{O}_{2}$ & 312.5 & $\begin{array}{l}\text { Antioxidant, } \\
\text { Hypocholesterolemic, } \\
\text { Antiandrogenic, } \\
\text { Hemolytic }\end{array}$ \\
\hline 9. & Ethyl tridecanoate & & $\mathrm{C}_{15} \mathrm{H}_{30} \mathrm{O}_{2}$ & 242.4 & Antibacterial, antifungal \\
\hline 10. & $\begin{array}{l}\text { 1H-3a, 7Methanoazulene, } \\
\text { octahydro-1,9,9-trimethyl- } \\
\text { 4methylene }\end{array}$ & & $\mathrm{C}_{15} \mathrm{H}_{24}$ & 204.4 & $\begin{array}{l}\text { Anti-inflammatory, } \\
\text { antiprotozoal,antibacterial } \\
\text { and antimicrobial }\end{array}$ \\
\hline 11. & $\begin{array}{l}\text { 1,4-Methanocycloocta[d] } \\
\text { pyridazine, } 1,4,4 \mathrm{a}, 5,6,9,10,10 \mathrm{a}- \\
\text { Octahydro-1 } 1,11 \text { dimethyl }\end{array}$ & & $\mathrm{C}_{20} \mathrm{H}_{30} \mathrm{O}$ & 286.5 & $\begin{array}{l}\text { Anti } \\
\text { allergicantidepressive, } \\
\text { antihypertensive, } \\
\text { antiinflammatory }\end{array}$ \\
\hline 12. & $\begin{array}{l}\text { Cycloprop[e]indene-1 a,2(1H)- } \\
\text { dimethanol,3a,4,5,6,6a,6b- } \\
\text { hexahydro-5,5,6b-trimethyl }\end{array}$ & & $\mathrm{C}_{15} \mathrm{H}_{24} \mathrm{O}_{2}$ & 236.4 & Anti-inflammatory \\
\hline 13. & $\begin{array}{l}\text { 1-Methoxy-5-methyl-5-phenyl- } \\
\text { 7-oxabicyclo[4. }\end{array}$ & & $\mathrm{C}_{18} \mathrm{H}_{23}$ & 317.4 & Antitubercular agent \\
\hline
\end{tabular}




\begin{tabular}{|c|c|c|c|c|c|}
\hline S.No & Name of the compound & Structure & $\begin{array}{l}\text { Chemical } \\
\text { formula }\end{array}$ & $\begin{array}{l}\text { Molecular } \\
\text { weight }\end{array}$ & Medicinal properties \\
\hline 14. & Benzene, 1,2-bis-(1-buten-3yl)- & & $\mathrm{C}_{14} \mathrm{H}_{18}$ & 186.3 & Antineoplastic agent \\
\hline 15. & $\begin{array}{l}\text { Benzene,(2-ethyl-4-methyl-1,3- } \\
\text { pentadienyl)-, }\end{array}$ & & $\mathrm{C}_{14} \mathrm{H}_{18}$ & 186.3 & $\begin{array}{l}\text { Antineoplastic agent, } \\
\text { antiviral, washing hair }\end{array}$ \\
\hline 16. & 1-Heptacosanol & & $\mathrm{C}_{27} \mathrm{H}_{56} \mathrm{O}$ & 396.7 & $\begin{array}{l}\text { Used against skin } \\
\text { cancer, antioxidant, } \\
\text { anticancer, antimalarial }\end{array}$ \\
\hline 17. & $\begin{array}{l}3 \text { beta-Hydroxy-5-cholen-24-oic } \\
\text { acid }\end{array}$ & & $\mathrm{C}_{24} \mathrm{H}_{38} \mathrm{O}_{3}$ & 374.6 & $\begin{array}{l}\text { Agreegation behavior, } \\
\text { cholesterol dissolution, } \\
\text { proliferation and } \\
\text { apoptosis in colan }\end{array}$ \\
\hline 18. & Stigmasterol & & $\mathrm{C}_{29} \mathrm{H}_{48} \mathrm{O}$ & 412.7 & $\begin{array}{l}\text { Antioxidant, } \\
\text { hypoglycemic, } \\
\text { antidiabetic, anti- } \\
\text { inflammatory }\end{array}$ \\
\hline 19. & $(+)-$ Lariciresinol & & $\mathrm{C}_{20} \mathrm{H}_{24} \mathrm{O}_{6}$ & 360.4 & Antifungal \\
\hline 20. & Obtusifoliol & & $\mathrm{C}_{30} \mathrm{H}_{50} \mathrm{O}$ & 426.7 & $\begin{array}{l}\text { Conjuctivitis, eye } \\
\text { disease, blurred vision, } \\
\text { asthma, ascaris }\end{array}$ \\
\hline 21. & $\begin{array}{l}\text { Androstan-17-one, 3-ethyl-3- } \\
\text { hydroxy-, (5.alpha) }\end{array}$ & & $\mathrm{C}_{21} \mathrm{H}_{34} \mathrm{O}_{2}$ & 318.5 & $\begin{array}{l}\text { Hypocholesterolemic, } \\
\text { nimeticide, pesticide }\end{array}$ \\
\hline 22. & $\begin{array}{l}\text { 3.beta-Hydroxy-5-cholen-24-oic } \\
\text { acid }\end{array}$ & & $\mathrm{C}_{24} \mathrm{H}_{40} \mathrm{O}_{3}$ & 376.6 & $\begin{array}{l}\text { Hepatoprotective, fat } \\
\text { absorption, cholesterol } \\
\text { excretion }\end{array}$ \\
\hline 23. & $\begin{array}{l}\text { 14-Oxatricyclo }[9.2 \cdot 1.0(1,10)] \\
\text { tetradecane, } 2,6,6,10,11- \\
\text { pentamethyl }\end{array}$ & & $\mathrm{C}_{18} \mathrm{H}_{30}$ & 262.4 & $\begin{array}{l}\text { Wound healing, } \\
\text { antiscarring }\end{array}$ \\
\hline 24. & beta-Amyrin & & $\mathrm{C}_{30} \mathrm{H}_{50} \mathrm{O}$ & 426.7 & $\begin{array}{l}\text { Antidiabetic, anti- } \\
\text { inflammatory, } \\
\text { anticancer }\end{array}$ \\
\hline 25 . & $\begin{array}{l}\text { 9,19-Cyclo-9.beta-lanostane- } \\
\text { 3.beta,25-diol }\end{array}$ & & $\mathrm{C}_{30} \mathrm{H}_{52} \mathrm{O}_{2}$ & 444.7 & $\begin{array}{l}\text { Hypoglycemic, } \\
\text { antidiabetic }\end{array}$ \\
\hline
\end{tabular}




\begin{tabular}{|c|c|c|c|c|c|}
\hline S.No & Name of the compound & Structure & \begin{tabular}{|l|} 
Chemical \\
formula \\
\end{tabular} & $\begin{array}{l}\text { Molecular } \\
\text { weight }\end{array}$ & Medicinal properties \\
\hline 26. & $\begin{array}{l}(1 \mathrm{~S}, 6 \mathrm{R}, 9 \mathrm{~S})-5,5,9,10- \\
\text { Tetramethyltricyclo[7.3.0 }\end{array}$ & & $\mathrm{C}_{12} \mathrm{H}_{18} \mathrm{Br}_{6}$ & 641.7 & $\begin{array}{l}\text { Antiinflammatory, } \\
\text { antibiotic,aiding } \\
\text { memory }\end{array}$ \\
\hline 27. & Piperidinone & & $\mathrm{C}_{5} \mathrm{H}_{9} \mathrm{NO}$ & 99.1 & Antifungal \\
\hline 28. & Tridecanoic acid 12-methyl ester & & $\mathrm{C}_{15} \mathrm{H}_{30} \mathrm{O}_{2}$ & 242.4 & No activity reported \\
\hline 29. & Calcitriol & & $\mathrm{C}_{27} \mathrm{H}_{44} \mathrm{O}_{3}$ & 416.7 & $\begin{array}{l}\text { Hyperparathyroidism, } \\
\text { absorption of calcium } \\
\text { from the stomach }\end{array}$ \\
\hline 30. & $\begin{array}{l}2,6,8 \text {-Trimethylbicyclo[4.2.0]oct- } \\
2 \text {-ene-1,8-dio }\end{array}$ & Br & $\mathrm{C}_{13} \mathrm{H}_{13} \mathrm{~N}_{5} \mathrm{~S}_{5} \mathrm{O}_{2}$ & 383.4 & Healing property \\
\hline 31. & 1,Z-5,E-7-Dodecatriene & & $\mathrm{C}_{12} \mathrm{H}_{20}$ & 164.3 & $\begin{array}{l}\text { Antitumour, } \\
\text { antibacterial, skin } \\
\text { disease }\end{array}$ \\
\hline 32. & $\begin{array}{l}\text { 8-Azabicyclo[3,2,1]octan-3-ol, 8- } \\
\text { methyl-, endo- }\end{array}$ & & $\mathrm{C}_{7} \mathrm{H}_{13} \mathrm{NO}$ & 127.2 & Antimuscarinic property \\
\hline 33. & Alfa-Copaene & & $\mathrm{C}_{15} \mathrm{H}_{24}$ & 204.4 & $\begin{array}{l}\text { Antioxidant, } \\
\text { antiproliferative, } \\
\text { antimicrobial }\end{array}$ \\
\hline 34. & $\begin{array}{l}\text { 1'-Vinyl-1'-2'-3'-4'- } \\
\text { tetrahydrospiro[1,3]dioxolar }\end{array}$ & & $\mathrm{C}_{4} \mathrm{H}_{6} \mathrm{O}$ & 70.1 & $\begin{array}{l}\text { Antidiabetic, Increased } \\
\text { immunity, hypoglycemic }\end{array}$ \\
\hline 35. & Phthalic acid & & $\mathrm{C}_{8} \mathrm{H}_{6} \mathrm{O}_{4}$ & 166.1 & $\begin{array}{l}\text { Synthesis of dyes, } \\
\text { perfumes and other } \\
\text { organic compound }\end{array}$ \\
\hline 36. & $\begin{array}{l}\text { 1-Butanamine, 3-methyl-N-(3- } \\
\text { methylbutalide }\end{array}$ & & $\mathrm{C}_{7} \mathrm{H}_{14} \mathrm{O}_{2}$ & 130.2 & $\begin{array}{l}\text { Antioxidant, prevent } \\
\text { heart disease, antidote } \\
\text { for cyanide poisoning }\end{array}$ \\
\hline 37. & Butanedioicacid,monomethyl ester & & $\mathrm{C}_{5} \mathrm{H}_{8} \mathrm{O}_{4}$ & 132.1 & $\begin{array}{l}\text { A molecular entity } \\
\text { capable of donating a } \\
\text { hydron acceptor }\end{array}$ \\
\hline 38. & $\begin{array}{l}\text { 1-(+)-Lactic acid, tert- } \\
\text { butyldimethylsilyl ester }\end{array}$ & & $\mathrm{C}_{15} \mathrm{H}_{34} \mathrm{O}_{3} \mathrm{Si}_{2}$ & 318.6 & Prevent skin disease \\
\hline 39. & $\begin{array}{l}\text { 5-Isopropylidene-4, 6- } \\
\text { dimethylnona-3,6,8-trien- }\end{array}$ & & $\mathrm{C}_{14} \mathrm{H}_{22}$ & 206.3 & $\begin{array}{l}\text { Hypertension, cough, } \\
\text { headache }\end{array}$ \\
\hline
\end{tabular}




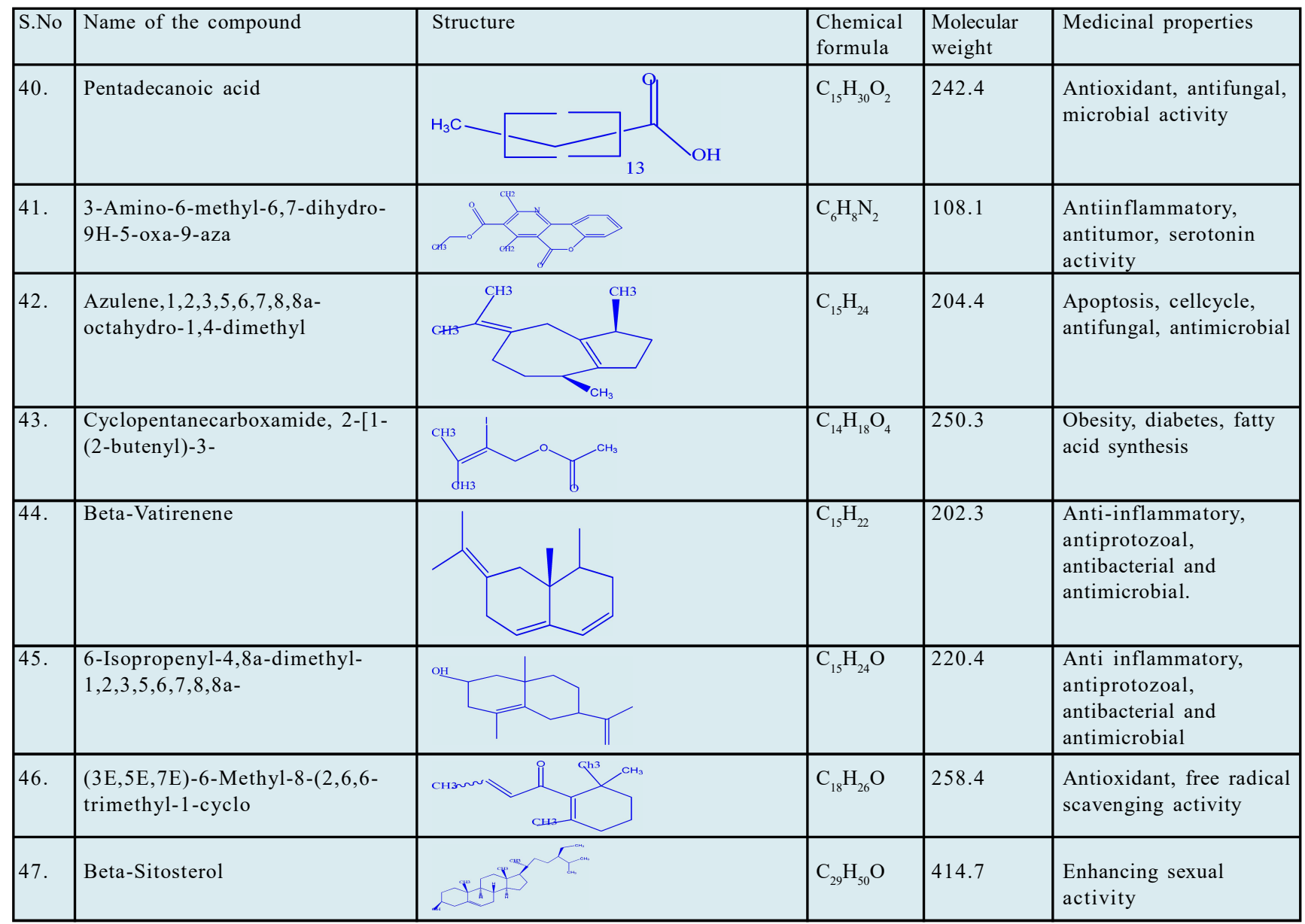

- The structure, molecular weight and medicinal properties of resolved compounds are compiled from the available literature.

\section{Discussion}

T. cordifolia is a plant with very high medicinal value against a large number of ailments (Sharma et al., 2014; Mishra et al., 2013; Saha and Ghosh, 2012). Researchers across the globe have developed great interest in this plant because of many of its reported medicinal activities like antineoplastic, anti-HIV, anticancer, hepatoprotective, immunomodulatory, antileprotic, antistress, antiarthritic, antiallergic, antidiabetic, antispasmodic, anti-inflammatory, etc. A large number of bioactive phytocomponents, viz., alkaloids, steroids, diterpenoid lactones, aliphatics, and glycosides have been isolated from all parts of the plant including root, stem or the whole plant (Kavitha et al., 2011; Ahmad et al., 2010). For extraction of phytochemicals from T. cordifolia in the present study, water, ethanol and methanol were selected which are commonly used solvents for extraction. Many secondary metabolites including phenolics, alkaloids, glycosides, flavanoids and carbohydrates are soluble in methanol while ethanol is good solvent for extraction of alkaloids, saponins, tannins, phenolics, glycosides, proteins and amino acids. Methanol and ethanol are neither too toxic nor too volatile (B.P. $64.7^{\circ} \mathrm{C}$ and $78.4^{\circ} \mathrm{C}$, respectively). Mostly methanol and ethanol are used for extraction of various polar compounds while certain non-polar compounds are also soluble in these solvents (polarity indices of methanol and ethanol are 6.6 and 5.2, respectively) (IIoki-Assango et al., 2015).

Different groups of compounds have been detected in all the three solvents used for extraction including alkaloids, flavonoids, glycosides, carbohydrates, proteins, tannins and phenols. These secondary metabolites are very useful compounds being extensively used in medicine as anticancer, in heart diseases, analgesics, antimalarials, antibiotic, antibacterial, etc., neutraceuticals, antidotes for reducing poisoning's by toxic compounds, in research as metabolic inhibitors for tracing various pathways, etc. Highest amount of alkaloids were detected only in ethanolic extract while proteins, carbohydrates, tannins and phenols are present in higher amounts in aqueous extract when compared with ethanolic or methanolic extracts of T. cordifolia while flavonoids were present only in aqueous extract. The presence of wide range of phytochemicals of medicinal importance in different species of Tinospora has been compiled earlier also (Chi et al., 2016). The starch obtained from the stem, is highly nutritive and digestive and is used in many diseases. Starch is present throughout the parenchyma of the stem (Sharma et al., 2013). It is a tonic, useful in the treatment of dysentery, chronic diarrhea and other diseases of 
gastrointestinal tract and protects the liver from damage by various drugs and chemicals. This is especially useful when the liver has been exposed to various toxins. Results indicate the presence of many phyto-components in the extracts prepared in all three solvents. These compounds may contribute the activities like antioxidant, cancer-preventive, hypocholesterolemic, nematicidal, antifungal, antidiabetic, hepatoprotective, etc. Hence, the stem of T. cordifolia is worthy for further investigation for being used in natural drug developments.

Beta amyrin present in all the three extracts is reported for its antidiabetic properties (Singh et al., 2009). The other compounds reported for their antidiabetic properties are 1-vinyl1, 2, 3, 4-tetrahydrospiro [1,3] dioxolar extracted in water, cyclopentanecarboxamide, 2-[1-(2-butenyl)-3-in ethanol and 9,19cyclo-9.betalanostane-3.beta,25-diol and stigmasterol in methanol. Hexadecanoic acid methyl ester, ethyl tridecanoate and heptacosanol were common in aqueous and methanolic extracts of $T$. cordifolia, 2,6,8-Trimethylbicyclo[4.2.0] oct-2-ene-1,8-dio was common in aqueous and ethanolic extracts, $1 \mathrm{H}-3 \mathrm{a}, 7-$ Methanoazulene, octahydro-1,9,9-trimethyl, 1,4,4a,5,6,9,10,10aoctahydro-11,11-dimethyl and 1,4-Methanocycloocta[d] pyridazine, were common in ethanolic and methanolic extracts of T. cordifolia. Androstan present in all three extracts, is known for hypocholesterolemic and nematicidal properties, whereas hexadecanoic present in aqueous extract and methanolic extracts is reported for antioxidant, hypocholesterolemic and pesticide properties, ethyl tridecanoate is known for antibacterial and antifungal properties (Arora and Saini, 2017), hepatocosanol for skin cancer, antioxidant, anticancer and antimalarial properties, whereas 2,6,8 trimethylbicyclo[4,2,0] oct-2-ene-1,8-dio present in aqueous extract and ethanolic extracts is known for healing properties as reported by Bowler et al. (2001). In our earlier work, we have identified that aqueous extract of $T$. cordifolia showed highest antidiabetic property as compared to other extract (Dubey and Srivastava, 2017). Therefore,1'-Vinyl-1, 2, 3, 4-tetrahydrospiro $[1,3]$ dioxolar present only in aqueous extract along with beta amyrin may be responsible for the antidiabetic properties. Other compounds present in aqueous extracts are reported to have antibacterial, antifungal, anticancer, antioxidant, anti-inflammatory, hyperthyroidism, absorption of calcium from the stomach, antimuscarinic, antiproliferative, antidiabetic, immune-stimulatory and hypoglycemic properties as well as being used in the synthesis of dyes, perfumes, and other organic compounds as reported by several workers earlier. Remaining compounds present in ethanolic extract are reported to have anti-inflammatory, antibacterial, antimicrobial, antiallergic, antidepressant, antioxidant, prevent heartache, serotonin activity, cell cycle, apoptosis, obesity, fatty acid synthesis, antiprotozoal, free radical scavenging activity, enhancing sexual activity, etc. (Kanthal et al., 2014). The compounds present in methanolic extracts showed antimicrobial, antioxidant, anti-inflammatory, pesticide, hypocholesterolemic, antispasmodic, immunomodulatory, emulsifying agent, soaps, paint, hemolytic, antiandrogenic, antitubercular agent, aggregation behavior, proliferation, blurred vision, asthma, antiascaris, hepatoprotective, fat absorption and memory enhancing properties. Presence of a large number of compounds showing various activities in all the three extracts has been listed in Table 4. The medicinal properties of the compounds present in all the three extracts of $T$. cordifolia stem have been compiled from the reported information and listed in Table 5 .

The stem of $T$. cordifolia contains many important phytochemical components such as alkaloids, carbohydrates, flavonoids, etc. In FTIR analysis, aqueous and ethanolic extracts contained 11 peaks while methanolic extract showed 10 peaks, confirming the presence of compounds with at least 11 and 10 types of functional groups, respectively. In GC-MS analysis, the methanolic extract is found to be the richest in active components as maximum number of phytoconstituents have been extracted in this solvent. Compounds with reported antidiabetic and hepatoprotective effects have been isolated from all the three extracts of $T$. cordifolia, however in our earlier report, aqueous extract showed highest antidiabetic and hepatoprotective properties compared with other two extracts (Dubey and Srivastava, 2017). Knowledge of chemical constituents of plant is important and desirable because such information will be useful for synthesis of new chemical compounds with greater therapeutic efficacy.

\section{Conclusion}

Results of the present study have shown the presence of various phytocomponents in all the extracts prepared in water, ethanol and methanol. Methanol seems to be the best solvent for extraction of such components as highest number of compounds have been extracted in this solvent. The antidiabetic and antioxidant compounds has been found widely distributed in all the three extracts with water being slightly superior solvent which is of benefit due to its safety. Further, validation of isolated compounds for their antihyperglycemic potential is mandatory for their use as antidiabetic agent for humans. Further, investigations and testing of the isolated compounds from T. cordifolia extracts is required which may become a part of standard drug designing and treatment protocols for diabetes and hence a promising and powerful weapon for diabetes treatment.

\section{Acknowledgements}

The help and support of Dr. V. Rajkumar, Senior Scientist, GPT Division, CIRG Makhdoom, Farah, Mathura 281122, India, in carrying out GC-MS analysis and Mr. Virendra Shankhwar, Technician, Central Instrumentation Facility, Jiwaji University, Gwalior-474011, India in carrying out FTIR-analysis are thankfully acknowledged. The authors are thankful to the Department of Science and Technology, New Delhi, India, for FIST Grant (Grant \# SR/ST/LS1-152/2003) to the School.

\section{Conflict of interest}

The authors declare that there are no conflicts of interest in the course of conducting the research. All the authors had final decision regarding the manuscript and agreed to submit findings for publication. 


\section{References}

Adeoye-Isijola, M.O.; Olajuyigbe, O.O.; Jonathan, S.G. and Coopoosamy, R.M (2018). Bioactive compounds in ethanol extract of lentinus squarrosulusmont: A Nigerian medicinal macrofungus. Afi. J. Tradit. Compl. Alternat. Med., 15(2):42-50.

Adhvaryu, M.R.; Reddy, N. and Parabia, M.H. (2007). Effects of four Indian medicinal herbs on Isoniazid-Rifampicin-and Pyrazinamideinduced hepatic injury and immunosuppression in guinea pigs. World J. Gastroenterol., 13(23):3199-3205.

Ahmad, F.; Ali, M. and Alam, P. (2010). New phytoconstituents from the stem bark of Tinospora cordifolia Miers. Nat. Prod. Res., 24(10):926-934.

Ahn, K. (2017). The worldwide trend of using botanical drugs and strategies for developing global drugs. BMC Rep., 50(3):111-116.

Albinjose, A.; Jasmine, E.; Selvankumar, T. and Srinivasakumar, K.P. (2015). Bioactive compounds of Tinospora cordifolia by gas chromatographymass spectrometry (GC-MS). Int. J. Multidis. Res. Dev, 2(1):88-97.

Arora, S. and Saini, M. (2017). Gas chromatography mass spectrometry profiling in methanolic and ethyl-acetate roots and stem extract of Corbichonia decumbens (Forssk.) Exell from Thar desert of Rajasthan India. Pharmacogonosy Res., 9(1): 548-552.

Balandrin, M.F.; Klocke, J.A.; Wurtele, E.S. and Bollinger, W.H. (1985). Natura plant chemicals: Sources of industrial and medicinal materials. Science, 228(4704):1154-1160.

Balunas, M.J. and Kinghorn, A.D. (2005). Drug discovery from medicinal plants. Life Sci., 78(1):431-441.

Bowler, P.G.; Duerden, B.I. and Armstrong, D.G. (2001). Wound microbiology and associated approaches to wound management. Clin. Microbiol. Rev., 14(2):244-269

Chi, S.; She, G.; Han, D.; Wang, W.; Liu, Z. and Liu, M. (2016). Genus Tinospora Ethanophaomacology, phytochemistry and pharmacology. Evid. Based Complement. Alternat. Med., Article ID; 9232593.

Dhama, K.; Sachan, S.; Khandia, R.; Munjal, A.; Iqbal, H.M.N.; Lateef, S.K.; Karthik, K.; Samad, H.A.; Tiwari, R. and Dadar, M. (2017). Medicinal and beneficial health applications of Tinospora cordifolia (Guduchi): A meraculous herb countering various diseases/disorders and its immunomodulatory effects. Recent Pat. Endocr. Metab. Immune. Drug Discov., 10(1):96-111.

Dhandapani, R. and Babna, B. (2008). Phytochemical constituents of some Indian medicinal plants. Anc. Sci. Life, 27(4):1-8.

Dubey, P. and Srivastava, N. (2017). Evaluation of hepatoprotective potential of Tinospora cordifolia extracts in fructose - induced experimental diabetic rats. Int. J. Pharma. Bio. Sci., 8(3):830-838.

Ekor, M. (2014). The growing use of herbal medicines: issues relating to adverse reactions and challenges in monitoring safety. Frontiers Pharmacol., 4(1):1-10.

Ezeonu C.S. and Ejikeme C.M. (2016). Qualitative and quantitative determination of phytochemical contents of indigenous nigerian softwoods. New J. Science, Article ID 5601327 | https://doi.org/ $10.1155 / 2016 / 5601327$

Foulger, J.H. (1931). The user of the molisch ( $\alpha$-Naphthol) reactions in the study of sugars in biological fluids. J. Biol. Chem., 92:345-353. http://en.wikipedia.org/wiki/ Biuret_test
IIoki-Assanga, S.B.; Lewis-Lujan, L.M.; Lara- Espinoza, C.L.; Gil-Salido, A.A.; Fernandez-Augulo, D.; Rubio-Pino, J.L. and Haines, D.D. (2015). Solvent effects on phytochemical constituent profiles and antioxidant activities using four different extraction formulation for analysis of Bucida buceras L. and Phoradendron californicum. BMC Res. Notes, 8(1):396-409.

Jaiswal, Y.S. and Williams, L.L. (2016). A glimpse of Ayurveda: The forgotten history and principles of Indian traditional medicine. J. Tradit. Complement. Med., 7(1):50-53.

Kanthal, L.K.; Dey, A. and Bhojaraju, P. (2014). GC-MS analysis of bio-active compound in methanolic extract of Lactucarun cinata DC. Pharmacognosy Res., 6(1):58-61.

Karmi, A.; Majlesi, M. and Rafieian-Kopaei, M. (2015). Herbal versus synthetic drugs; beliefs and facts. J. Nephropharmacol., 4(1):2730 .

Kaur, G.; Prabhakar, P.K.; Lal, U.R. and Suttee, A. (2009). Phytochemical and biological analysis of Tinospora cordifolia. Int. J. Toxicol. Pharmacol. Res., 8(4):297-305.

Kavitha, B.T.; Shruthi, S.D.; Rai, S.P. and Ramachandra, Y.L. (2011). Phytochemical analysis and hepatoprotective properties of Tinospora cordifolia against carbon tetrachloride-induced hepatic damage in rats. J. Basic. Clin. Pharm., 2(3):139-142.

Khan, J.A. and Srivastava, R. (2012). Study on antibacterial property of Tinospora cordifolia leaf, stem and root. The Int. J. Pharm. Res. BioSci., 1(6):291-298.

Lee, S.E.; Hwang, H.J.; Ha, J.S.; Jeong, H.S. and Kim, J.H. (2013). Screening of medicinal plant extracts for antioxidant activity. Life. Sci., 1(2): $167-179$

Maobe, M.A.G. and Nyarango, R.M. (2013). Fourier transformer infra-red spectrophotometer analysis of urticadioica medicinal herb used for the treatment of diabetes, malaria and pneumonia in kisii region, Southwest Kenya, ID: 14550751.

Mishra, A.; Kumar, S. and Pandey, A.K. (2013). Scientific Validation of the Medicinal Efficacy of Tinospora cordifolia. Sci. World J., Article ID: 292934

Mosihuzzaman, M. (2012). Herbal medicine in healthcare: An overview. Nat. Prod. Commun., 7(6):1154-12.

National Medical Plants Board, Ministry of AYUSH (2016). Government of India, http://nmpb.nic.in/2016.

Pan, S.Y.; Litscher, G.; Gao, S.H.; Zhou, S.F.; Yu, Z.L.; Chen, H.Q.; Zhang, S.F.; Tang, M.K.; Sun, J.N. and Ko, K.M. (2014). Historical perspective of traditional indigenous medical practices: The current renaissance and conservation of herbal resources. Evid. Based Complement. Alternat. Med., 2014:525340.doi: 10.1155/2014/525340.

Panchabhai, T.S.; Kulkarni, U.P. and Rege, N.N. (2008). Validation of therapeutic claims of Tinospora cordifolia: A review. Phytother. Res., 22(4):425-441.

Rates, S.M. (2001). Plants as source of drugs. Toxicon., 39(5):603-613.

Robert, W, (1965). Siegfried ruhemann and the discovery of ninhydrin. J. Chem. Education, 42(7):386-388. doi: 10.1021/ed042p386.

Saha, S. and Ghosh, S. (2012). Tinospora cordifolia: One plant, many roles. Anc. Sci. Life, 31(4):151-159.

Sharma, R., Galib and Prajapati, P.K. (2014). Remarks on Tinospora cordifolia: One plant, many roles. Anc. Sci. Life, 33(3):194. 
Sharma, R.; Amin, H.; Galib, R. and Prajapati, P.K. (2013). Seasonal variations in physicochemical profiles of Guduchi Satva (starchy substance from Tinospora cordifolia [Willd. Miers). J. Ayurveda Integr. Med., 4(4):193-197.

Singh, A.B.; Yadav, D.K.; Maurya, R. and Srivastava, A.K. (2009). Antihyperglycemic activity of alpha-amyrin acetate in rats and db/db mice. Nat. Prod. Res., 23(9):876-882.

Sofowora, A.; Ogunbodede, F. and Onayade, O. (2013). The role and place of medicinal plants in the strategies for disease prevention. Afr. J. Tradit. Complement. Altern. Med., 10(5):210-229.
Tao, G.; Wei-Hong, F.; Xiao-Qian, L.; Hui-Min., W. and Liang-Liang, G. (2015). Fourier transform mid-infrared spectroscopy (FT-MIR) combined with chemometrics for quantitative analysis of dextrin in Danshen (Salvia miltiorrhiza) granule. J. Pharm. Biomed. Anal., 123(1):16-23.

Vaidya, A.K. and Devasagayam, T.P.A. (2007). Current status of herbal drugs in India: An overview. J. Clin. Biochem. Nutr., 41(1):1-11.

WHO (2005). National policy on traditional medicine regulation of herbal medicines. Report of a World Health Organization Global Survey, Geneva, Switzerland: WHO, 2005.

Zhao, T.F.; Wang, X.K. and Rimando, A.M. (1991). Folkloric medicinal plants: Tinospora sagittata var. cravaniana and Mahonia bealei. Planta Med., 57(5):505.

Citation Preety Dubey, Shiv Kumar Jayant and Nalini Srivastava (2020). Preliminary phytochemical screening, FTIR and GC-MS analyses of aqueous, ethanolic and methanolic extracts of stem of Tinospora cordifolia (Guduchi) for search of antidiabetic compounds. Ann. Phytomed., 9(2):183-197. http://dx.doi.org/10.21276/ap.2020.9.2.16 